\title{
Highly Excited Core Resonances in Photoionization of Fe XVII : Implications for Plasma Opacities
}

\author{
Sultana N. Nahar ${ }^{1}$, Anil K. Pradhan ${ }^{1}$, Guo-Xin Chen $^{2}$, Werner Eissner ${ }^{3}{ }^{*}$ \\ ${ }^{1}$ Department of Astronomy, The Ohio State University, Columbus, Ohio 43210, \\ 2 ITAMP, Harvard-Smithsonian Center for Astrophysics, 60 Garden Street, Cambridge, \\ MA 02138, ${ }^{3}$ Institut für Theoretische Physik, Teilinstitut 1, 70550 Stuttgart, Germany
}

(Dated: November 15, 2018)

\begin{abstract}
A comprehensive study of high-accuracy photoionization cross sections is carried out using the relativistic Breit-Pauli R-matrix (BPRM) method for $(\mathrm{h} \nu+\mathrm{Fe}$ XVII $\rightarrow$ Fe XVIII $+\mathrm{e})$. Owing to its importance in high-temperature plasmas the calculations cover a large energy range, particularly the myriad photoexciation-of-core (PEC) resonances including the $n=3$ levels not heretofore considered. The calculations employ a close coupling wave function expansion of 60 levels of the core ion $\mathrm{Fe}$ XVIII ranging over a wide energy range of nearly $900 \mathrm{eV}$ between the $n=2$ and $n=3$ levels. Strong coupling effects due to dipole transition arrays $2 p^{5} \rightarrow 2 p^{4}(3 s, 3 d)$ manifest themselves as large PEC resonances throughout this range, and enhance the effective photoionization cross sections orders of magnitude above the background. Comparisons with the erstwhile Opacity Project (OP) and other previous calculations shows that the currently available cross sections considerably underestimate the bound-free cross sections. A level-identification scheme is used for spectroscopic designation of the 454 bound fine structure levels of Fe XVII , with $n \leq 10, l \leq 9$, and $0 \leq J \leq 8$ of even and odd parities, obtained using the ab initio BPRM method (compared to 181 LS bound states in the OP work). The calculated energies are compared with those available from the National Institute for Standards and Technology database, which lists 63 levels with very good agreement. Level-specific photoionization cross sections are computed for all levels. In addition, partial cross sections for leaving the core ion Fe XVII in the ground state are also obtained. These results should be relevant to modeling of astrophysical and laboratory plasma sources requiring (i) photoionization rates, (ii) extensive non-local-thermodynamic-equilibrium models, (iii) total unified electron-ion recombination rates including radiative and dielectronic recombination, and (iv) plasma opacities. We particularly examine PEC and non-PEC resonance strengths and emphasize their expanded role to incorporate inner-shell excitations for improved opacities, as shown by the computed monochromatic opacity of Fe XVII .
\end{abstract}

PACS numbers: 32.80.Fb,96.60.Jw,52.70.-m

\section{INTRODUCTION}

High precision studies of photoionization of atoms in a large number of excited levels are of interest in several areas. Derivative quantities such as laboratory and astrophysical plasma opacities [1-4], spectral models of photoionization dominated soures such as active galactic nuclei [5], non-local-thermodynamic-equilibrium models of stellar atmospheres [6], and total electron-ion recombination rates [7], depend on the accuracy of underlying treatment of photoionization for the entire atom(ion) at all energies where it is abundant under speciffic plasma conditions.

There have been a number of theoretical calculations to produce large amounts of data for practical applications, in particular under the Opacity Project (OP) [3] and the Iron Project (IP) 32]. But they are usually limited in terms of accuracy owing to computational constraints, and in detailed examination of the photoabsorption process for excited levels. Furthermore, except for light elements or simple few-electron systems, large-

*Electronic address: nahar@astronomy.ohio-state.edu scale calculations do not adequately treat the two most important atomic effects, relativistic fine structure and resonances. Many previous calculations are basically in LS coupling [3], or which take account of fine structure through an algebraic transformation rather than a relativistic calculation per se. The primary reason of course is the difficulty of doing so, since the calculations for photoionization of a large number of fine structure levels remains an enormous task. Close coupling calculations that account for the ubiquitous resonances in photoionization cross sections are mostly done using the R-matrix method [8-10]. The extension to include relativistic effects led to the development of the Breit-Pauli R-matrix (BPRM) codes [11, 12], and to aforementioned work under the Iron Project for relatively simple atomic systems such as the He-like and Li-like ions (e.g. [13]).

Such large-scale calculations with high precision may be of crucial importance in resolving a major astrophysical problem [14] that is related to plasma opacities [15] and recent studies of solar abundances of common elements such as C, N, O and Ne. The new abundances are widely discordant with the standard solar abundances by up to $50 \%$ 14]. The new abundances are derived from high-resolution spectroscopy and the most advanced 3D hydrodynamic non-local-thermodynamic-equilibrium 
(NLTE) models However, such a huge decrease in solar elemental composition is not supported by heliosmological observations of solar oscillations measured with great accuracy [16]. In addition, predictions of solar interior models are also in conflict with the new abundances [17]. Given the inverse relationship between opacity and abundances, tt has been suggested that a marginal, but highly significant, increase in mean opacities of about 10-20\% could possible reconcile the new and the standard solar abundances [18].

Another need for higher precision opacities arises due to the recent capability of creating stellar interior conditions in the laboratory [1]. Inertial confinement fusion (ICF) devices can now produce plasmas in LTE at temperatures and densities approaching those at the boundary of the radiative zone and the convection zone $(\mathrm{CZ})$ in the Sun. Measurements have been carried out at the Z-pinch facility at the Sandia National Laboratory of monochromatic opacity spectra of iron at $\mathrm{T}_{e} \sim 150-200$ $\mathrm{eV}$ and $\mathrm{N}_{e} \sim 10^{22-23} / \mathrm{cc}$. Fe XVII is the largest contributor to opacity amongst the iron ions prevalent at that temperature and density, from Fe XVI - Fe XIX with ionization fractions $0.0 .0757,0.302,0.366$ and 0.18 respectively. However, there are marked differences between the Z-pinch observations and theoretically computed opacities [1]. Thus, both astrophysical and laboratory observations require high-precision studies to understand or resolve the discrepancies.

We now note one of the basic atomic physics assumptions made in earlier theoretical calculations of plasma opacities: resonances are treated as lines, and perturbative treatments are then employed to include continuum contribuions. Depending of the temperature and density, most of the opacity could stem from inner-shell excitations in complex atomic species such as iron ions with closed L and M subshells [2, 19]. Such photoexcitationof-core (PEC) process occurs at high energies above the first ionization threshold. Therefore, it manfiests itself as resonances in the bound-free. The corresponding PEC resonances are large features present at incident photon frequencies corresponding to dipole transitions in the core ion [23, 27]. It follows that the major source of opacity in any plasma source, astrophysical or in the laboratory, lies in bound-free resonances in general, heretofore treated mostly as bound-bound transitions or lines, and in PEC resonances in particular. But the approximation of resonant photoionization by lines was made in the $\mathrm{OP}$ [2, 3] and OPAL [4] projects because opacities calculations are extremely intensive, and require huge amounts of atomic data for all consituent elements in many excitation/ionization states. Nevertheless, good agreement is found between the two projects for mean opacities over a wide range of temperatures and densities (19], discussed later). But now, owing to the major problems outlined above, it appears necessary to compute atomic parameters to higher precision to address this issue, albeit on a relatively small scale for individiual ions to begin with.

This report presents pilot and prototypical calculations required for revised opacities work. Ne-like Fe XVII is a prime contributor to solar opacity near the convection zone boundary [1], with many PEC features in photoionization cross sections. By carrying out one of the most comprehensive BPRM calculations for photoionization of an atomic system, we have computed and analyzed levelspecific fine structure cross sections, and the distribution of resonances over a wide energy range encompassing complex atomic structures. In previous studies of photoionization including resonances, Fe XVII was studied mainly in the low energy region using a small wavefunction representation. An R-matrix calculation in LS coupling using a 2-state wave function expansion for the core ion Fe XVIII was carried out under the Opacity Project (M. P. Scott, see [39]). A later coupled channel calculation, aimed at unfied electron-ion recombination calculations for $(\mathrm{e}+\mathrm{Fe}$ XVIII $) \rightarrow$ Fe XVII , employed the (BPRM) method using a 3-level (or 3CC) wave function expanion by [20]. The recombination spectrum obtained using $3 \mathrm{CC}$ photoionization compared very well with the experimentally observed spectrum in the very low energy region. But due to the ion's abundance in high temperature plasmas, a preliminary calculation using a 60-level expansion for Fe XVIII was initiated by [21]. This initial study on photoionization cross sections focused only on one symmetry: levels with total $J=0$. But to examine its opacity in toto a much more extensive calculation is necessary. This study is a full-scale photoionization calculation using the large 60-level wave function expansion for Fe XVIII, with energy levels up to approximately $1 \mathrm{keV}$. That energy range should account for nearly all of the resonance structures due to L-shell excitation of Fe XVII at temperatures $\sim 200 \mathrm{eV}$ where it is abundant near the solar CZ; the Planckian blackbody distribution decreases to render Fe XVII ionization fraction and opacity to be insignifiant beyond $\mathrm{E} \sim 1.5 \mathrm{keV}$. We especially aim to elucidate photoexcitation-of-core (PEC) resonance features that greatly affect photoionization in the high energy region.

In this report we point out other potential applications as well. Since the cross sections are computed up to levels with $n(S L J) \leq 10$, they should be useful for non-LTE spectral modeling in the X-ray region. The present work yields accurate energies and spectroscopic identification of the 454 bound levels of Fe XVII computed through ab initio calculations. In addition, level-specific partial photoionization cross sections of Fe XVII into the ground state of Fe XVIII are computed, as required for future studies of unified recombination cross sections including both radiative and dielectronic recombination processes.

\section{THEORY}

Photoionization calculations have been carried out in the close coupling (CC) approximation using the Rmatrix method as developed under the Opacity Project [2, 3] and the Iron Project [12, 32]. In the CC approxima- 
tion the atomic system is represented as the 'target' or the 'core' ion of N-electrons interacting with the $(\mathrm{N}+1)^{t h}$ electron. The $(\mathrm{N}+1)^{t h}$ electron may be bound in the electron-ion system, or in the electron-ion continuum depending on its energy to be negative or positive. The total wavefunction, $\Psi_{E}$, of the $(\mathrm{N}+1)$-electron system in a symmetry $J \pi$ is an expansion over the eigenfunctions of the target ion, $\chi_{i}$ in specific state $S_{i} L_{i}\left(J_{i}\right) \pi_{i}$, coupled with the $(\mathrm{N}+1)^{t h}$ electron function, $\theta_{i}$ :

$$
\Psi_{E}(e+i o n)=A \sum_{i} \chi_{i}(i o n) \theta_{i}+\sum_{j} c_{j} \Phi_{j}
$$

where the sum is over the ground and excited states of the target or the core ion. The $(\mathrm{N}+1)^{t h}$ electron with kinetic energy $k_{i}^{2}$ corresponds to a channel labelled $S_{i} L_{i}\left(J_{i}\right) \pi_{i} k_{i}^{2} \ell_{i}(S L(J) \pi)$. The $\Phi_{j} \mathrm{~s}$ are bound channel functions of the $(\mathrm{N}+1)$-electron system that account for short range correlation not considered in the first term and the orthogonality between the continuum and the bound electron orbitals of the target.

The relativistic Hamiltonian in the Breit-Pauli Rmatrix (BPRM) approximation is given by

$$
\begin{aligned}
& H_{N+1}^{\mathrm{BP}}= \\
& \sum_{i=1}^{N+1}\left\{-\nabla_{i}^{2}-\frac{2 Z}{r_{i}}+\sum_{j>i}^{N+1} \frac{2}{r_{i j}}\right\}+H_{N+1}^{\operatorname{mass}}+H_{N+1}^{\mathrm{Dar}}+H_{N+1}^{\mathrm{so}} .
\end{aligned}
$$

where the last three terms are relativistic corrections, respectively:

the mass correction term, $H^{\text {mass }}=-\frac{\alpha^{2}}{4} \sum_{i} p_{i}^{4}$, the Darwin term, $H^{\text {Dar }}=\frac{Z \alpha^{2}}{4} \sum_{i} \nabla^{2}\left(\frac{1}{r_{i}}\right)$, the spin - orbit interaction term, $H^{\text {so }}=Z \alpha^{2} \sum_{i} \frac{1}{r_{i}^{3}} \mathbf{l}_{\mathbf{i}} \cdot \mathbf{s}_{\mathbf{i}}$.

Eq.(3) representes the one-body terms of the Breit interaction. Another version of "full" BPRM codes has been developed including the two-body terms, but those are only of importance in forbidden (E2, M1, M2) transitions where two-electrons correlations may play an important role [25]. In contrast, plasma opacities in toto are determined by strong E1 (dipole allowed and intercombination) considered in this work.

Substitution of $\Psi_{E}(e+i o n)$ in the Schrodinger equation

$$
H_{N+1} \Psi_{E}=E \Psi_{E}
$$

introduces a set of coupled equations that are solved using the R-matrix method. The solution is a continuun wavefunction $\Psi_{F}$ for an electron with positive energies (E $>0$ ), or a bound state $\Psi_{B}$ at a negative total energy ( $\mathrm{E} \leq$ $0)$. The complex resonance structures in photoionization cross sections result from channel couplings between the continuum channels that are open $\left(k_{i}^{2}>0\right)$, and ones that are closed $\left(k_{i}^{2}<0\right)$. Resonances occur at electron energies $k_{i}^{2}$ corresponding to autoionizing states belonging to Rydberg series, $S_{i} L_{i} \pi_{i} \nu \ell$ where $\nu$ is the effective quantum number, converging on to the target threshold $S_{i} L_{I}$.
The first term on the right in Eq. (1) represents both bound states and free (continuum) states of the $(e+i o n)$ system. If all channels are closed then the state is bound an represented by $\Psi_{B}$; asymptotically, all channel functions are expnentially decaying. On the other hand, a continuum state corresponds to some channels open and some closed, and is referred to as $\Psi_{F}$. Asymptotically, the open channel functions are oscillating, as for a free electron. The transition matrix element for photoionization is

$$
<\Psi_{B}\|\mathbf{D}\| \Psi_{F}>
$$

where $\mathbf{D}=\sum_{i} r_{i}$ is the dipole operator and the sum is over the number of electrons; $\Psi_{B}$ and $\Psi_{F}$ are the bound and continuum wave functions. The transition matrix element can be reduced to generalized line strength as

$$
\mathbf{S}=\mid\left\langle\Psi_{j}|| \mathbf{D}_{L}|| \Psi_{i}>\left.\right|^{2}=\left|\left\langle\psi_{f}\left|\sum_{j=1}^{N+1} r_{j}\right| \psi_{i}\right\rangle\right|^{2},\right.
$$

where $\Psi_{i}$ and $\Psi_{f}$ are the initial and final state wave functions. The photoionization cross section $\left(\sigma_{P I}\right)$ is proportional to the generalized line strength as,

$$
\sigma_{P I}=\frac{4 \pi^{2}}{3 c} \frac{1}{g_{i}} \omega \mathbf{S},
$$

where $g$ is the statistical weight factor of the bound state and $\omega$ is the incident photon energy (Ry).

\section{COMPUTATIONS}

BPRM photoionization cross sections of Fe XVII were computed in the 60CC expansion over the core ion $\mathrm{Fe}$ XVIII , and free-electron wave functions with partial waves up to $l \leq 12$. The one-electron basis set of orbitals used to construct a configuration-interaction representation of the eigenfunctions of Fe XVIII were obtained from the code SUPERSTRUCTURE [29], which employs a Thomas-Fermi-Dirac-Amaldi central field potential. The choice of the one-electron orbital basis set is not unique; Hartree-Fock orbitals of Slater type orbitals are also used in R-matrix calculations [8 10]. At sufficiently high densities, viz. $N_{e}>10^{22} \mathrm{cc}$, the electronic orbitals may themselves be altered by plasma effects such as Debye screening. However, we do not consider those effects in OP calculations which entail the isolated atom approximation and the so called "chemical picture" 2, 33] (discussed later).

Table 1 gives the set of 11 configurations with filled $1 \mathrm{~s}^{2}$ shell that was optimized to obtain Fe XVIII core wave functions [21]. It lists the 60 levels included in the $60 \mathrm{CC}$ expansion. For a small number of levels the available energy level data [26] compiled by the National Institute for Standards and Technology (NIST), is found to be in very good agreement with theoretical calculations [21]. 
TABLE I: Energy levels of the target ion Fe XVIII included in the eigenfunction expansion of Fe XVII . Note the large energy gap of $\sim 47$ Ry between the $n=2$ and $n=3$ complexes. The target was optimized with a set of 11 configurations with open L-shell but closed K-shell: $2 \mathrm{~s}^{2} 2 \mathrm{p}^{5}(1)$, $2 \mathrm{~s} 2 \mathrm{p}^{6}(2), 2 \mathrm{~s}^{2} 2 \mathrm{p}^{4} 3 \mathrm{~s}(3), 2 \mathrm{~s}^{2} 2 \mathrm{p}^{4} 3 \mathrm{p}(4), 2 \mathrm{~s}^{2} 2 \mathrm{p}^{4} 3 \mathrm{~d}(5), 2 \mathrm{~s} 2 \mathrm{p}^{5} 3 \mathrm{~s}(6)$, $2 \mathrm{~s} 2 \mathrm{p}^{5} 3 \mathrm{p}(7), 2 \mathrm{~s} 2 \mathrm{p}^{5} 3 \mathrm{~d}(8), 2 \mathrm{p}^{6} 3 \mathrm{~s}(9), 2 \mathrm{p}^{6} 3 \mathrm{p}(10), 2 \mathrm{p}^{6} 3 \mathrm{~d}(11)$.

\begin{tabular}{|c|c|c|c|c|c|c|c|c|c|}
\hline $\mathrm{i}$ & $\begin{array}{l}\text { Config- } \\
\text { uration }\end{array}$ & Term & $2 J$ & $\begin{array}{r}E(\mathrm{Ry}) \\
\text { Present }\end{array}$ & $\mathrm{i}$ & $\begin{array}{l}\text { Config- } \\
\text { uration }\end{array}$ & Term & $2 J$ & $\begin{array}{c}E(\text { Ry }) \\
\text { Present }\end{array}$ \\
\hline \multicolumn{5}{|c|}{$\mathrm{n}=2$ states } & 30 & $2 s^{2} 2 p^{4} 3 p$ & ${ }^{2} P^{o}$ & 1 & 61.899 \\
\hline 1 & $2 s^{2} 2 p^{5}$ & ${ }^{2} P^{o}$ & 3 & 0.00000 & 31 & $2 s^{2} 2 p^{4} 3 d$ & ${ }^{4} D$ & 5 & 62.299 \\
\hline 2 & $2 s^{2} 2 p^{5}$ & ${ }^{2} P^{o}$ & 1 & 0.93477 & 32 & $2 s^{2} 2 p^{4} 3 d$ & ${ }^{4} D$ & 7 & 62.311 \\
\hline 3 & $2 s 2 p^{6}$ & ${ }^{2} S$ & 1 & 9.70228 & 33 & $2 s^{2} 2 p^{4} 3 d$ & ${ }^{4} D$ & 1 & 62.906 \\
\hline \multicolumn{5}{|c|}{$\mathrm{n}=3$ states } & 34 & $2 s^{2} 2 p^{4} 3 d$ & ${ }^{4} D$ & 3 & 63.050 \\
\hline 4 & $2 s^{2} 2 p^{4} 3 s$ & ${ }^{4} P$ & 5 & 56.690 & 35 & $2 s^{2} 2 p^{4} 3 p$ & ${ }^{2} P^{o}$ & 3 & 62.461 \\
\hline 5 & $2 s^{2} 2 p^{4} 3 s$ & ${ }^{2} P$ & 3 & 56.936 & 36 & $2 s^{2} 2 p^{4} 3 d$ & ${ }^{4} F$ & 9 & 62.535 \\
\hline 6 & $2 s^{2} 2 p^{4} 3 s$ & ${ }^{4} P$ & 1 & 57.502 & 37 & $2 s^{2} 2 p^{4} 3 d$ & ${ }^{2} F$ & 7 & 62.629 \\
\hline 7 & $2 s^{2} 2 p^{4} 3 s$ & ${ }^{4} P$ & 3 & 57.572 & 38 & $2 s^{2} 2 p^{4} 3 p$ & ${ }^{2} P^{o}$ & 1 & 62.686 \\
\hline 8 & $2 s^{2} 2 p^{4} 3 s$ & ${ }^{2} P$ & 1 & 57.798 & 39 & $2 s^{2} 2 p^{4} 3 d$ & ${ }^{4} P$ & 1 & 62.496 \\
\hline 9 & $2 s^{2} 2 p^{4} 3 s$ & ${ }^{2} D$ & 5 & 58.000 & 40 & $2 s^{2} 2 p^{4} 3 d$ & ${ }^{4} P$ & 3 & 62.625 \\
\hline 10 & $2 s^{2} 2 p^{4} 3 s$ & ${ }^{2} D$ & 3 & 58.355 & 41 & $2 s^{2} 2 p^{4} 3 d$ & ${ }^{4} F$ & 5 & 62.985 \\
\hline 11 & $2 s^{2} 2 p^{4} 3 p$ & ${ }^{4} P^{o}$ & 3 & 59.209 & 42 & $2 s^{2} 2 p^{4} 3 d$ & ${ }^{2} P$ & 1 & 63.123 \\
\hline 12 & $2 s^{2} 2 p^{4} 3 p$ & ${ }^{4} P^{o}$ & 5 & 59.238 & 43 & $2 s^{2} 2 p^{4} 3 d$ & ${ }^{4} F$ & 3 & 63.156 \\
\hline 13 & $2 s^{2} 2 p^{4} 3 p$ & ${ }^{4} P^{o}$ & 1 & 59.478 & 44 & $2 s^{2} 2 p^{4} 3 d$ & ${ }^{2} F$ & 5 & 62.698 \\
\hline 14 & $2 s^{2} 2 p^{4} 3 p$ & ${ }^{4} D^{o}$ & 7 & 59.525 & 45 & $2 s^{2} 2 p^{4} 3 d$ & ${ }^{4} F$ & 7 & 63.271 \\
\hline 15 & $2 s^{2} 2 p^{4} 3 p$ & ${ }^{2} D^{o}$ & 5 & 59.542 & 46 & $2 s^{2} 2 p^{4} 3 d$ & ${ }^{2} D$ & 3 & 63.302 \\
\hline 16 & $2 s^{2} 2 p^{4} 3 s$ & ${ }^{2} S$ & 1 & 59.916 & 47 & $2 s^{2} 2 p^{4} 3 d$ & ${ }^{4} P$ & 5 & 62.911 \\
\hline 17 & $2 s^{2} 2 p^{4} 3 p$ & ${ }^{2} P^{o}$ & 1 & 59.982 & 48 & $2 s^{2} 2 p^{4} 3 d$ & ${ }^{2} P$ & 3 & 63.308 \\
\hline 18 & $2 s^{2} 2 p^{4} 3 p$ & ${ }^{4} D^{o}$ & 3 & 60.005 & 49 & $2 s^{2} 2 p^{4} 3 d$ & ${ }^{2} D$ & 5 & 63.390 \\
\hline 19 & $2 s^{2} 2 p^{4} 3 p$ & ${ }^{4} D^{o}$ & 1 & 60.012 & 50 & $2 s^{2} 2 p^{4} 3 d$ & ${ }^{2} G$ & 7 & 63.945 \\
\hline 20 & $2 s^{2} 2 p^{4} 3 p$ & ${ }^{2} D^{o}$ & 3 & 60.147 & 51 & $2 s^{2} 2 p^{4} 3 d$ & ${ }^{2} G$ & 9 & 63.981 \\
\hline 21 & $2 s^{2} 2 p^{4} 3 p$ & ${ }^{4} D^{\circ}$ & 5 & 60.281 & 52 & $2 s^{2} 2 p^{4} 3 d$ & ${ }^{2} S$ & 1 & 63.919 \\
\hline 22 & $2 s^{2} 2 p^{4} 3 p$ & ${ }^{2} P^{o}$ & 3 & 60.320 & 53 & $2 s^{2} 2 p^{4} 3 d$ & ${ }^{2} F$ & 5 & 64.200 \\
\hline 23 & $2 s^{2} 2 p^{4} 3 p$ & ${ }^{2} S^{o}$ & 1 & 60.465 & 54 & $2 s^{2} 2 p^{4} 3 d$ & ${ }^{2} F$ & 7 & 64.301 \\
\hline 24 & $2 s^{2} 2 p^{4} 3 p$ & ${ }^{4} S^{\circ}$ & 3 & 60.510 & 55 & $2 s^{2} 2 p^{4} 3 d$ & ${ }^{2} P$ & 3 & 64.138 \\
\hline 25 & $2 s^{2} 2 p^{4} 3 p$ & ${ }^{2} F^{o}$ & 5 & 60.851 & 56 & $2 s^{2} 2 p^{4} 3 d$ & ${ }^{2} D$ & 5 & 64.160 \\
\hline 26 & $2 s^{2} 2 p^{4} 3 p$ & ${ }^{2} F^{o}$ & 7 & 61.028 & 57 & $2 s^{2} 2 p^{4} 3 d$ & ${ }^{2} D$ & 3 & 64.391 \\
\hline 27 & $2 s^{2} 2 p^{4} 3 p$ & ${ }^{2} D^{o}$ & 3 & 61.165 & 58 & $2 s^{2} 2 p^{4} 3 d$ & ${ }^{2} P$ & 1 & 64.464 \\
\hline 28 & $2 s^{2} 2 p^{4} 3 p$ & ${ }^{2} D^{o}$ & 5 & 61.272 & 59 & $2 s^{2} 2 p^{4} 3 d$ & ${ }^{2} D$ & 5 & 65.305 \\
\hline 29 & $2 s^{2} 2 p^{4} 3 p$ & ${ }^{2} P^{o}$ & 3 & 61.761 & 60 & $2 s^{2} 2 p^{4} 3 d$ & ${ }^{2} D$ & 3 & 65.468 \\
\hline
\end{tabular}

One of the main points to note, and which is of considerable significance in the energy distribution of resonances, is the clustering of 60 fine structure levels in the $n=2$ and $n=3$ complexes, and the large energy gap of $\sim 50$ Ry between them. Although the target energies are quite accurate, some further improvement in the positions of resonances is also achieved by replacing the Fe XVIII level energies with observed ones, wherever available [26], during diagonalization of the $(\mathrm{N}+1)$-electron Hamiltonian of the $(\mathrm{e}+\mathrm{Fe}$ XVIII $)$ system.

The second sum in the wavefunction expansion given in Eq. (1) is the bound-channel term consisting of selected electronic configurations for the electron-ion system. We include 27 configurations of $(N+1)$-electron bound channels of Fe XVII , specified by a range of mininum and maximum occupancies (listed within parentheses after the orbitals) as: $2 s(0-2), 2 p(3-6), 3 s(0-2), 3 p(0-2)$ and $3 d(0-2)$. All $S L J \pi$ symmetries of the electron-ion system formed from the target states coupled with an interacting electron with continnum partial waves $0 \leq l \leq$ 12 are considered.

The ab initio bound state energies of the electron-ion system computed by the R-matrix codes in intermediate
$S L J$ coupling are not spectroscopically identified a priori. For complex ions it is a highly non-trivial task to assign $L S$ term and $S L J$ level designations. It is particularly difficult for fine structure levels computed in BPRM calculations owing to near-degeneracy of levels in high- $Z$ or high- $z$ ions, particularly for high- $n$ and $l$. The code PRCBPD [37] is employed for level identification, using the information computed by the BPRM codes, as explained in the next section. In addition, the BPRM energies are cross checked against observations and SUPERSTRUCTURE whereever possible. However, we still note that this is one of the most laborious tasks requiring some judgement in the final assignments for levels highly mixed by configuration interaction.

The present calculations cover a large energy range and variations in the distribution of resonance complexes. In the near-threshold region, below $n=2$ levels, the $\sigma_{P I}$ were resolved on a fine energy mesh, with 4000 energies upto 0.4 Ry above the ionization threshold. However, such fine resolution is computationally prohibitive for all symmetries and levels over the entire range of $\sim 65 \mathrm{Ry}$ where resonances due to L-shell excitation occur. There are energy regions where the resonances are sparse, as opposed to regions where they are densely clustered. A number of energy meshes are used to ensure that the overall, as well as the detailed, contribution of resonances to $\sigma_{P I}$ is taken into account. At photoelectron energies above all 60 target thresholds, the $\sigma_{P I}$ are extrapolated as in [36].

\section{RESULTS AND DISCUSSION}

The results of Fe XVII are divided into a few subsections below.

\section{A. Fine Structure Levels and Oscillator Strengths of Fe XVII}

Present BPRM calculations for fine structure bound levels are intended to form a complete set for most practical applications. As explained in an earlier paper [34], the high-lying excited core states do not form bound states of the electron-ion system, and corresponding channels have insignificant effect on the bound state energies. This is particularly true of multiply ionized ions, where there are large gaps between the ground complex and the next excited complex of levels; the energy separation increases as $z r$. The core ion Fe XVIII has an energy gap of $\sim 47 \mathrm{Ry}$ between the $n=2$ and $n=3$ levels (Table 1). Although the present study includes both the $n=2$ and 3 complexes, all bound levels of Fe XVII have the Fe XVIII parent level as the ground level or another $n=2$ excited level, i.e. $2 s^{2} p^{5}{ }^{2} P_{1 / 2,3 / 2}^{o}, 2 s 2 p^{6}{ }^{2} S_{1 / 2}$.

R-matrix calculations for bound energy levels entail a 'search' for zeroes of the electron-ion Hamiltonian 
[32, 38], where the eigenvalues of the $(\mathrm{N}+1)$-electron system occur as in Eq. (4). A total of 454 eigenenergies of $\mathrm{Fe}$ XVII were found, subject to the choice of $n \leq 10,0 \leq l \leq$ 9 , and $0 \leq J \leq 8$ of even and odd parities. These levels have been identified spectroscopically using a numerical procedure implemented in the code PRCBPID [37]. It is based on (i) detailed analysis of quantum defects along Rydberg series of levels, (ii) parentage of the core ion states, (iii) fractional or percentage contribution of closed channels which translates into configuration interaction of corresponding bound electron-ion configurations, and (iv) angular momentum algebra. The level and energies are similar to the $3 \mathrm{CC}$ case carried [21], with some differences because of mixed levels and near-degenerate quantum defects.

For relatively few levels the present calculated energies of Fe XVII are compared with the observed values available in the NIST compilation [26] in Table 2. The overall agreement is very good, $\sim 0.1 \%$ or better, for most levels including ones that are highly excited. The number next to the $J$-value in Tabel 2 (Col. 3 ) is the relative position of the correspoding calculated energy in its own symmetry $J \pi$. As an example we discuss levels of a particular summetry $S L J:{ }^{1} P_{1}^{o}$. In the present work we obtain $35{ }^{1} P_{1}^{o}$ levels, as opposed to 11 given in the NIST tables; they are compared in Table 2. Of those 11 energies, 5 energies agree to $\sim 0.1 \%$ or better. That includes highly excited levels such as $2 s^{2} 2 p^{6} 4 p{ }^{1} P_{1}^{o}$, listed as the $19^{\text {th }}$ computed level (Col. 3). For two other levels, $2 s^{2} 2 p^{5} 6 d^{1} P_{1}^{o}$ and $2 s^{2} 2 p^{5} 5 d^{1} P_{1}^{o}$, the energies agree to $\sim$ $0.2 \%$ and $0.3 \%$ respectively. However, the energies of the remaining three levels differ by $1-4 \%$. The largest discrepancy is for the level $2 s^{2} 2 p^{5} 5 s^{1} P_{1}^{o}$. The complete set of 454 energies with spectroscopic designations is available electronically (at the NORAD atomic data website given at the end of the paper).

We also report that the oscillator strengths for electric dipole transitions among all the bound levels are obtained and are available electronically. The earlier set corresponds to 3-CC calculations [41].

\section{B. Photoionization Cross Sections of Fe XVII}

Photoionization cross sections of all 454 bound fine structure levels of Fe XVII are computed and analyzed in a variety of ways, in particular the distribution of resonances that lie in the large energy gap between the $n$ $=2$ and $n=3$ complexes. This is a comprehensive set of cross sections computed using the BPRM method, as required for applications such as plasma opacities, synthetic spectral models, and spectral diagnostics of X-ray absorption lines observed from astronomical objects. The BPRM calculations yield about two and a half times as many levels as the previous OP calculations in LS coupling [39], which resulted in 181 LS bound states with $n(S L) \leq 10$. Some important charateristic features of the BPRM cross sections are illustrated and discussed
TABLE II: Comparison of calculated energies, $E_{c}$, of Fe XVII with the measured values, $E_{o}[26]$. $i_{J}$ indicates position of the calculated level for symmetry $J$. An asterisk next to a level indicates incomplete set of observed levels for the state.

\begin{tabular}{|c|c|c|c|c|}
\hline Conf & Term & $J: i_{J}$ & $E_{o}(\mathrm{Ry})$ & $E_{c}(\mathrm{Ry})$ \\
\hline $2 s^{2} 2 p^{6}$ & ${ }^{1} S$ & $0.0: 1$ & $9.2760 \mathrm{E}+01$ & $9.2925 \mathrm{E}+01$ \\
\hline $2 s^{2} 2 p^{5} 3 s$ & ${ }^{3} P^{o}$ & $2.0: 1$ & $3.9463 \mathrm{E}+01$ & $3.9503 \mathrm{E}+01$ \\
\hline $2 s^{2} 2 p^{5} 3 s$ & ${ }^{3} P^{o}$ & $1.0: 1$ & $3.9323 \mathrm{E}+01$ & $3.9367 \mathrm{E}+01$ \\
\hline $2 s^{2} 2 p^{5} 3 s$ & ${ }^{3} P^{o}$ & $0.0: 1$ & $3.8533 \mathrm{E}+01$ & $3.8560 \mathrm{E}+01$ \\
\hline $2 s^{2} 2 p^{5} 3 s$ & ${ }^{1} P^{o}$ & $1.0: 2$ & $3.8446 \mathrm{E}+01$ & $3.8469 \mathrm{E}+01$ \\
\hline $2 s^{2} 2 p^{5} 3 p$ & & $1.0: 1$ & $3.7238 \mathrm{E}+01$ & $3.7284 \mathrm{E}+01$ \\
\hline $2 s^{2} 2 p^{5} 3 p$ & ${ }^{3} D$ & $3.0: 1$ & $3.6863 \mathrm{E}+01$ & $3.6902 \mathrm{E}+01$ \\
\hline $2 s^{2} 2 p^{5} 3 p$ & ${ }^{3} D$ & $2.0: 1$ & $3.6981 \mathrm{E}+01$ & $3.7027 \mathrm{E}+01$ \\
\hline $2 s^{2} 2 p^{5} 3 p$ & ${ }^{3} D$ & $1.0: 3$ & $3.6093 \mathrm{E}+01$ & $3.6114 \mathrm{E}+01$ \\
\hline $2 s^{2} 2 p^{5} 3 p$ & ${ }^{1} P$ & $1.0: 2$ & $3.6780 \mathrm{E}+01$ & $3.6826 \mathrm{E}+01$ \\
\hline $2 s^{2} 2 p^{5} 3 p$ & ${ }^{3} P$ & $2.0: 2$ & $3.6646 \mathrm{E}+01$ & $3.6688 \mathrm{E}+01$ \\
\hline $2 s^{2} 2 p^{5} 3 p$ & ${ }^{3} P$ & $1.0: 4$ & $3.5854 \mathrm{E}+01$ & $3.5880 \mathrm{E}+01$ \\
\hline $2 s^{2} 2 p^{5} 3 p$ & ${ }^{3} P$ & $0.0: 2$ & $3.6244 \mathrm{E}+01$ & $3.6274 \mathrm{E}+01$ \\
\hline $2 s^{2} 2 p^{5} 3 p$ & ${ }^{1} D$ & $2.0: 3$ & $3.5826 \mathrm{E}+01$ & $3.5843 \mathrm{E}+01$ \\
\hline $2 s^{2} 2 p^{5} 3 p$ & ${ }^{1} S$ & $0.0: 3$ & $3.4871 \mathrm{E}+01$ & $3.4828 \mathrm{E}+01$ \\
\hline $2 s^{2} 2 p^{5} 3 d$ & ${ }^{3} P^{o}$ & $2.0: 2$ & $3.3662 \mathrm{E}+01$ & $3.3669 \mathrm{E}+01$ \\
\hline $2 s^{2} 2 p^{5} 3 d$ & ${ }^{3} P^{o}$ & $1.0: 3$ & $3.3778 \mathrm{E}+01$ & $3.3813 \mathrm{E}+01$ \\
\hline $2 s^{2} 2 p^{5} 3 d$ & ${ }^{3} P^{o}$ & $0.0: 2$ & $3.3862 \mathrm{E}+01$ & $3.3895 \mathrm{E}+01$ \\
\hline $2 s^{2} 2 p^{5} 3 d$ & ${ }^{3} F^{o}$ & $4.0: 1$ & $3.3656 \mathrm{E}+01$ & $3.3651 \mathrm{E}+01$ \\
\hline $2 s^{2} 2 p^{5} 3 d$ & ${ }^{3} F^{o}$ & $3.0: 1$ & $3.3599 \mathrm{E}+01$ & $3.3612 \mathrm{E}+01$ \\
\hline $2 s^{2} 2 p^{5} 3 d$ & ${ }^{3} F^{o}$ & $2.0: 4$ & $3.2672 \mathrm{E}+01$ & $3.2670 \mathrm{E}+01$ \\
\hline $2 s^{2} 2 p^{5} 3 d$ & ${ }^{1} D^{o}$ & $2.0: 3$ & $3.3472 \mathrm{E}+01$ & $3.3494 \mathrm{E}+01$ \\
\hline $2 s^{2} 2 p^{5} 3 d$ & ${ }^{3} D^{\circ}$ & $3.0: 2$ & $3.3393 \mathrm{E}+01$ & $3.3400 \mathrm{E}+01$ \\
\hline $2 s^{2} 2 p^{5} 3 d$ & ${ }^{3} D^{o}$ & $2.0: 5$ & $3.2598 \mathrm{E}+01$ & $3.2601 \mathrm{E}+01$ \\
\hline $2 s^{2} 2 p^{5} 3 d$ & ${ }^{3} D^{o}$ & $1.0: 4$ & $3.3052 \mathrm{E}+01$ & $3.3053 \mathrm{E}+01$ \\
\hline $2 s^{2} 2 p^{5} 3 d$ & ${ }^{1} F^{o}$ & $3.0: 3$ & $3.2563 \mathrm{E}+01$ & $3.2565 \mathrm{E}+01$ \\
\hline $2 s^{2} 2 p^{5} 3 d$ & ${ }^{1} P^{o}$ & $1.0: 5$ & $3.2070 \mathrm{E}+01$ & $3.2049 \mathrm{E}+01$ \\
\hline $2 s 2 p^{6} 3 p$ & ${ }^{3} P^{o}$ & $1.0^{*}: 6$ & $2.7159 \mathrm{E}+01$ & $2.7122 \mathrm{E}+01$ \\
\hline $2 s 2 p^{6} 3 p$ & ${ }^{1} P^{o}$ & $1.0: 7$ & $2.6836 \mathrm{E}+01$ & $2.6809 \mathrm{E}+01$ \\
\hline $2 s^{2} 2 p^{5} 4 s$ & ${ }^{3} P^{o}$ & $1.0^{*}: 8$ & $2.0899 \mathrm{E}+01$ & $2.0630 \mathrm{E}+01$ \\
\hline $2 s^{2} 2 p^{5} 4 s$ & ${ }^{1} P^{o}$ & $1.0: 9$ & $2.0014 \mathrm{E}+01$ & $2.0557 \mathrm{E}+01$ \\
\hline $2 s^{2} 2 p^{5} 4 d$ & ${ }^{3} P^{o}$ & $1.0^{*}: 10$ & $1.8802 \mathrm{E}+01$ & $1.8750 \mathrm{E}+01$ \\
\hline $2 s^{2} 2 p^{5} 4 d$ & ${ }^{3} D^{o}$ & $1.0^{*}: 11$ & $1.8455 \mathrm{E}+01$ & $1.8427 \mathrm{E}+01$ \\
\hline $2 s^{2} 2 p^{5} 4 d$ & ${ }^{1} P^{o}$ & $1.0: 12$ & $1.7590 \mathrm{E}+01$ & $1.7571 \mathrm{E}+01$ \\
\hline $2 s^{2} 2 p^{5} 5 s$ & ${ }^{3} P^{o}$ & $1.0^{*}: 13$ & $1.2960 \mathrm{E}+01$ & $1.2739 \mathrm{E}+01$ \\
\hline $2 s^{2} 2 p^{5} 5 s$ & ${ }^{1} P^{o}$ & $1.0: 14$ & $1.2022 \mathrm{E}+01$ & $1.2516 \mathrm{E}+01$ \\
\hline $2 s^{2} 2 p^{5} 5 d$ & ${ }^{3} P^{o}$ & $1.0^{*}: 15$ & $1.2022 \mathrm{E}+01$ & $1.1912 \mathrm{E}+01$ \\
\hline $2 s^{2} 2 p^{5} 5 d$ & ${ }^{3} D^{o}$ & $1.0^{*}: 16$ & $1.1776 \mathrm{E}+01$ & $1.1749 \mathrm{E}+01$ \\
\hline $2 s^{2} 2 p^{5} 5 d$ & ${ }^{1} P^{o}$ & $1.0: 17$ & $1.0910 \mathrm{E}+01$ & $1.0873 \mathrm{E}+01$ \\
\hline $2 s 2 p^{6} 4 p$ & ${ }^{3} P^{o}$ & $1.0^{*}: 18$ & $1.0236 \mathrm{E}+01$ & $1.0197 \mathrm{E}+01$ \\
\hline $2 s 2 p^{6} 4 p$ & ${ }^{1} P^{o}$ & $1.0: 19$ & $1.0090 \mathrm{E}+01$ & $1.0080 \mathrm{E}+01$ \\
\hline $2 s^{2} 2 p^{5} 6 s$ & ${ }^{3} P^{o}$ & $1.0^{*}: 20$ & $8.7776 \mathrm{E}+00$ & $8.7515 \mathrm{E}+00$ \\
\hline $2 s^{2} 2 p^{5} 6 d$ & ${ }^{3} P^{o}$ & $1.0^{*}: 22$ & $8.1488 \mathrm{E}+00$ & $8.1405 \mathrm{E}+00$ \\
\hline $2 s^{2} 2 p^{5} 6 d$ & ${ }^{1} P^{o}$ & $1.0^{*}: 24$ & $7.2558 \mathrm{E}+00$ & $7.2410 \mathrm{E}+00$ \\
\hline $2 s^{2} 2 p^{5} 7 s$ & ${ }^{3} P^{o}$ & $1.0 *: 25$ & $6.3810 \mathrm{E}+00$ & $6.3535 \mathrm{E}+00$ \\
\hline $2 s^{2} 2 p^{5} 7 d$ & ${ }^{3} P^{o}$ & $1.0 *: 26$ & $5.9709 \mathrm{E}+00$ & $6.0260 \mathrm{E}+00$ \\
\hline $2 s^{2} 2 p^{5} 7 d$ & ${ }^{1} P^{o}$ & $1.0 *: 29$ & $5.0232 \mathrm{E}+00$ & $5.0588 \mathrm{E}+00$ \\
\hline & ${ }^{3} P^{o}$ & $1.0^{*}: 32$ & $4.4582 \mathrm{E}+00$ & $4.5627 \mathrm{E}+00$ \\
\hline $2 s^{2} 2 p^{5} 8 d$ & ${ }^{1} P^{o}$ & $1.0^{*}: 35$ & $3.6016 \mathrm{E}+00$ & $3.6512 \mathrm{E}+00$ \\
\hline
\end{tabular}

below.

\section{Photoionization of the Ground State}

First we consider the ground state of Fe XVII . Fig. 1 presents photoionization cross sections $\sigma_{P I}$ of the ground state $2 s^{2} 2 p^{6}{ }^{1} S$. While panel (a) presents the cross section in LS coupling under the Opacity Project (M. P. Scott, see TOPbase data [39]), panels (b,c) present $\sigma_{P I}$ from the relativistic BPRM calculations; (b) presents the total cross section, and (c) presents partial cross section for leaving the core ion Fe XVIII in the ground state 

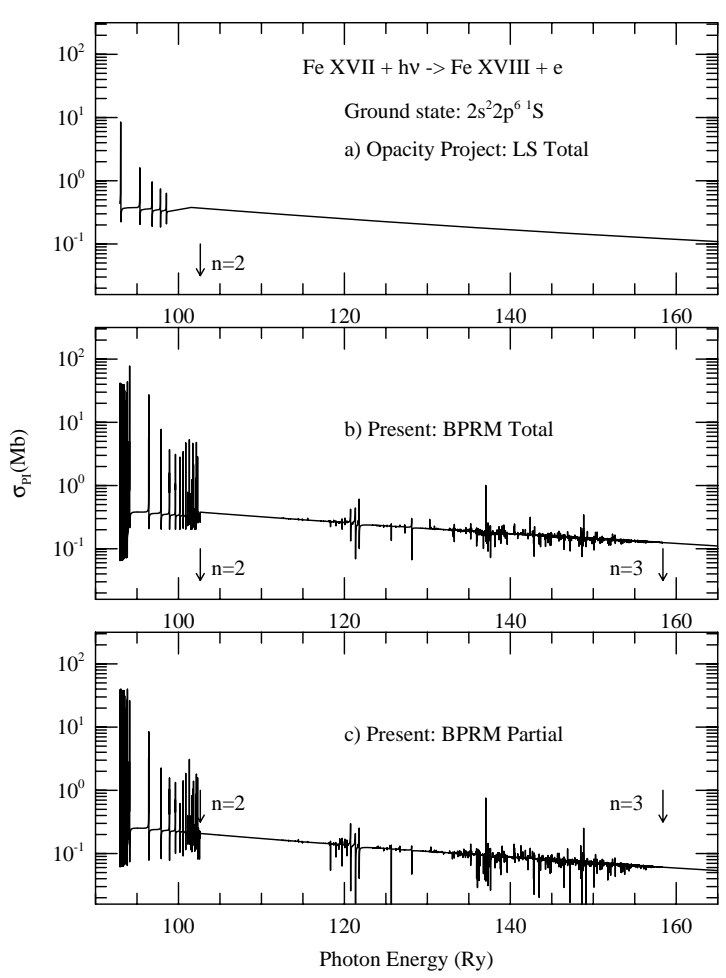

FIG. 1: Photoionization cross sections, $\sigma_{P I}$, of the ground level $1 s^{2} 2 s^{2} 2 p^{6}\left({ }^{1} S_{0}\right)$ of Fe XVII : (a) total $\sigma_{P I}$ in LS coupling from the Opacity Project database TOPbase [39], (b) present total $\sigma_{P I}$ in the relativistic BPRM formulation, (c) present partial $\sigma_{P I}$ with residual ion Fe XVIII in the ground state. Present calculations including relativistic fine structure provide more accurate energies and more highly resolved resonances. The background for the partial cross sections (c) is lower that the total (b) because the channels for leaving the core ion in various excited levels are excluded.

following photoionization. The earlier total $\sigma_{P I}$ from the two-state R-matrix OP calculation, and the present BPRM using a 60-level expansion are of similar magnitude for the background. But due to a much larger number of fine structure channels in the BPRM calculation, panel (b) shows many more resonances within the $n=2$ complex (as also found in the earlier 3CC calculation). Also, a much finer energy mesh in the present work has resolved the resonances more completely. Resolution of resonances in the low energy region, especially at and near threshold is crucial for calculating recombination and photoionization rates. The ground state $\sigma_{P I}$ at high energies decreases slowly, showing an insignificant effect due to highly excited $n=3$ core states in the $60 \mathrm{CC}$ calculation, except for introducing small and weak resonance structures.

The partial cross sections in panel (c) corresponds to photoionization of Fe XVII leaving the ionized core $\mathrm{Fe}$ XVIII only in the ground state. The background is lower than that of the total $\sigma_{P I}$ in (b) because there are no additional contributions from photoionization channels into excited levels. However the resonance features are the same for the total and the partial cross sections until the first excited state $2 s 2 p^{6}{ }^{1} S_{0}$ of Fe XVIII .

\section{Photoionization of Excited Levels}

Although the background ground state cross sections remain about the same between the $3 \mathrm{CC}$ and the $60 \mathrm{CC}$ calculations, the excited states are considerably more affected. One of the main results of the present BPRM calculations with a large wavefunction expansion is that photon absorptions and core excitations to the $n=3$ levels produce extensive resonance structures, in contrast to earlier calculations. This contradicts the assumption that channels of highly excited core states, especially when an energy gap like in the present case exists, might be too weakly interacting to produce any significant effect.

Fig. 2 presents total photoionization cross sections of excited levels $2 s^{2} 2 p^{5} 3 p\left({ }^{1} P_{1}^{o}\right)$ (a,b), and $2 s^{2} 2 p^{5} 3 d\left({ }^{1} D_{2}^{o}\right)$ $(\mathrm{c}, \mathrm{d})$. These two levels are chosen because they have a single fine structure component, so their cross sections can be directly compared with the earlier OP results for the LS terms $2 s^{2} 2 p^{5} 3 p\left({ }^{1} P^{o}\right)$ and $2 s^{2} 2 p^{5} 3 d\left({ }^{1} D^{o}\right)$. The top panels $(\mathrm{a}, \mathrm{c})$ of Fig. 2 present the OP results, and the bottom panels $(b, d)$ from the present work. Both of these excited levels show resonances due to core excitations to the $n=2$ and $n=3$ levels that are not present in the OP results. The features reveal how crucially important the set of resoances belonging to $n=3$ core thresholds are, compared to those of $n=2$. The resonance peaks are orders of magnitude higher than those from the $n=2$ series. There is only a relatively small gap with smooth background without resonances, between the highest threshold of the $n=2$ levels and the appearance of lowest of the $n=3$ complexes of resonances. While the background is enhanced considerably in the BPRM results by strong resonances, the featureless and slowly decreasing cross sections from the OP miss out all those features and vastly underestimate $\sigma_{P I}$.

Resonances in photoionization cross sections correspond to Rydberg series of autoionizing levels at energies $E_{\text {res }}=E_{c} \nu l$, where $E_{c}$ is an excited core threshold and $\nu_{l}$ are the efeective quantum number and angulur momentun of the interacting electron. The Rydberg resonances are narrow and lie below the threshold $E_{c}$, approximately at energies given by the simple expression

$$
E_{\text {res }}=E_{c}-z^{2} / \nu_{l}^{2}
$$

However, strong PEC resonances manfiest themselves in $\sigma_{P I}$ of excited bound levels of the electron-ion system [27, [35]. The PEC resonances are wide and occur at energies where the ground state of the core ion undergoes a strong dipole allowed transition. Among the 60 target 


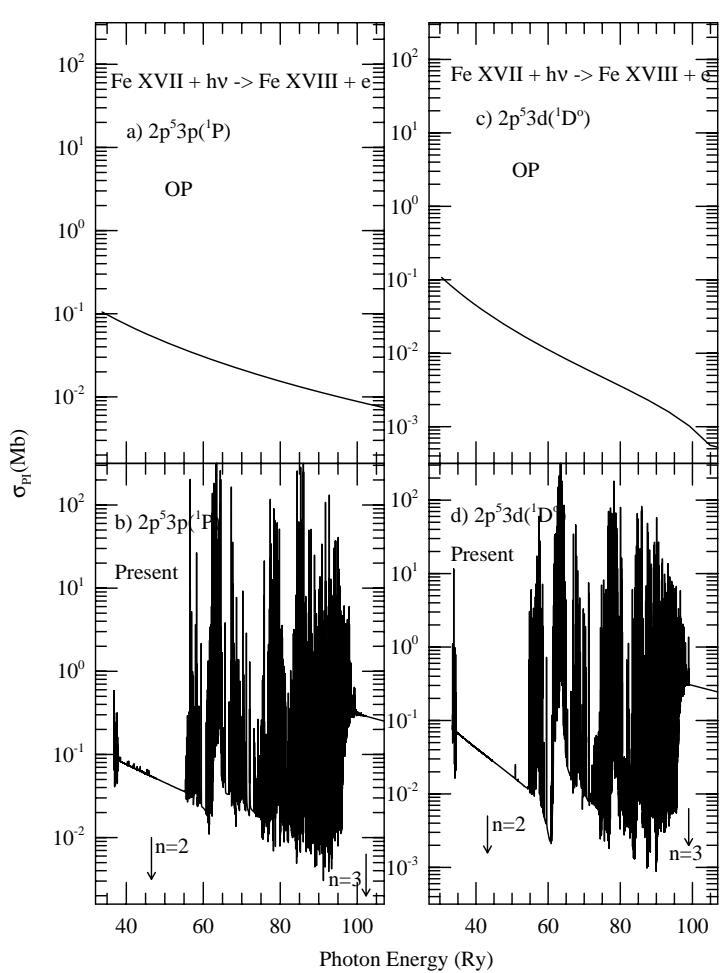

FIG. 2: Comparison of present photoionization cross sections $\sigma_{P I}$ with those from the OP (see [39]) for excited singlet levels (no fine structure): (a,b) $2 p^{5} 3 p^{1} P$, and (c,d) $2 p^{5} 3 d\left({ }^{1} D^{o}\right)$. In contrast to $\sigma_{P I}$ from $\mathrm{OP}$, the present results demonstrate that without inclusion of $n=3$ core levels of Fe XVIII the cross sections are considerably underestimated throughout most of the energy region of interest in practical applications. Resonances are included as lines in the OP work [3] , as in other opacities calculations [4].

or core levels for Fe XVIII there are 30 such transitions as given in Table 3 . Hence, in photoionization cross sections of Fe XVII there are 30 possible PEC resonances, most with overlapping profiles.

Fig. 3 presents photoionization cross sections of three highly excited levels of Fe XVII and demonstrably large PEC features, $2 s^{2} 2 p^{5} n f\left({ }^{3} D_{1}\right)$ with $n f=5 f, 7 f, 9 f$. Beyond the resonances due to $n=2$ core levels, the background decreases smoothly for the three levels. However, as the resonances due to $n=3$ core levels appear, the backgound rises and the PEC resonances manifest themselves as prominently high and wide structures. These enhancements are related to the radiative decay rates of the dipole allowed levels. Table 3 shows most of the decay rates from $n=3$ levels are much larger than those from the $n=2$ levels, by one or two orders of magnitude, and result in stronger resonances. With hundreds of bound levels computed in the present work, the cross
TABLE III: Dipole allowed and intercombination E1 transitions from the ground level $2 s^{2} 2 p^{5}\left({ }^{2} P_{3 / 2}^{o}\right)$ to excited states of the core ion Fe XVIII, and corresponding oscillator strengths $(f)$. These transitions introduce the PEC resonances in photoionization cross sections of Fe XVII . A number 'a(n)' means $\mathrm{a} \times 10^{n}$. The level indices correspond to those of Table 1 .

\begin{tabular}{|c|c|c|}
\hline & Tra & (PEC) \\
\hline & $8 p(13$ & \\
\hline & $2 s^{2} 2 p^{5}\left({ }^{2} P_{3}\right.$ & $57 \mathrm{E}-03$ \\
\hline & $2 s^{2} 2 p^{5}\left({ }^{2} P_{3 / 2}^{o}\right)-2 s^{2} 2 p^{4} 3 s\left({ }^{2} P\right.$ & $4.53 \mathrm{E}-02$ \\
\hline & $s^{2} 2 p^{5}\left({ }^{2} P_{3 / 2}^{o}\right)-2 s^{2} 2 p^{4} 3 s\left({ }^{2} P_{1 / 2}\right)$ & \\
\hline & $2 s^{2} 2 p^{5}\left({ }^{2} P_{3 / 2}^{o}\right)-2 s^{2} 2 p^{4} 3 s\left({ }^{4} P_{3 / 2}\right)$ & -02 \\
\hline & $2 s^{2} 2 p^{5}\left({ }^{2} P_{3 / 2}^{o}\right)-2 s^{2} 2 p^{4} 3 s\left({ }^{4} P_{1 / 2}\right)$ & \\
\hline & $2 s^{2} 2 p^{5}\left({ }^{2} P_{3 / 2}^{o}\right)-2 s^{2} 2 p^{4} 3 s\left({ }^{2} D_{5 / 2}\right)$ & \\
\hline & $2 s^{2} 2 p^{5}\left({ }^{2} P_{3 / 2}^{o}\right)-2 s^{2} 2 p^{4} 3 s\left({ }^{2} D_{3 / 2}\right)$ & \\
\hline & (3/2) $-2 s^{2} 2 p^{4} 3 s\left({ }^{2} S_{1 / 2}\right)$ & \\
\hline & $s^{2} 2 p^{4} 3 d\left({ }^{4} D_{5 / 2}\right)$ & \\
\hline & $2 s^{2} 2 p^{5}\left({ }^{2} P_{3 / 2}^{o}\right)$ & \\
\hline & $s^{2} 2 p^{4} 3 d\left({ }^{4} D_{1 / 2}\right)$ & \\
\hline & $s^{2} 2 p^{4} 3 d\left({ }^{4} P_{1 / 2}\right)$ & -05 \\
\hline & $s^{2} 2 p^{4} 3 d\left({ }^{4} P_{3 / 2}\right)$ & \\
\hline & $s^{2} 2 p^{4} 3 d$ & \\
\hline & $\left.{ }^{2} P_{1 / 2}\right)$ & \\
\hline & $2 s^{2} 2 p^{4} 3 d\left({ }^{2} D_{3 / 2}\right)$ & \\
\hline & $2 s^{2} 2 p^{4} 3 d\left({ }^{2} F\right.$ & \\
\hline & $2 s^{2} 2 p^{4} 3 d\left({ }^{4} F_{3}\right.$ & \\
\hline & $2 s^{2} 2 p^{4} 3 d\left({ }^{4} P\right.$ & -02 \\
\hline & $2 s^{2} 2 p^{4} 3 d\left({ }^{2} P\right.$ & \\
\hline & $-2 s^{2} 2 p^{4} 3 d\left({ }^{4} F_{5}\right.$ & \\
\hline & $2 s^{2} 2 p^{4} 3 d\left({ }^{2} F_{5 / 2}\right)$ & \\
\hline & $2 s^{2} 2 n^{4} 3 d c^{2} S$ & \\
\hline & $2 s^{2} 2 p^{4} 3 d\left({ }^{2} P_{3 / 2}\right)$ & \\
\hline & $d^{2} 2 n^{4} 3 d^{2} D$ & \\
\hline & ${ }^{2} 2 p^{4} 3 d\left({ }^{2} D_{3 / 2}\right)$ & \\
\hline & ${ }^{2} 2 p^{4} 3 d\left({ }^{2} P_{1 / 2}\right)$ & \\
\hline & $-2 s^{2} 2 p^{4} 3 d\left({ }^{2} D_{5 / 2}\right)$ & \\
\hline $1-60$ & $2 s^{2} 2 p^{5}\left({ }^{2} P_{3 / 2}^{o}\right)-2 s^{2} 2 p^{4} 3 d\left({ }^{2} D_{3 / 2}\right)$ & 3.72E-03 \\
\hline
\end{tabular}

sections in the high energy region from about $57 \mathrm{Ry}$ to $65 \mathrm{Ry}$ is greatly enhanced, mainly by complexes of PEC resonances corresponding to core excitations via dipole transitions listed in Table 3.

Of particular importance is the characterstic shape of PEC resonances. The resonances encompass an energy range of $\sim 10 \mathrm{Ry}$ or well over $100 \mathrm{eV}$. The shape is determined by channel coupling effects. In the case of the simple limit of an isolated resonance it is like the typical Fano profile. However, the PECs are generally affected by strong coupling among many target levels and channels, with a large number of superimposed non-PEC resonances converging on to the target levels. The distribution of the continuum or the differential oscillator strength would not be generally reproduced by an isolated resonance approximation, and requires a coupled channel calculation.

\section{Resonance oscillator strengths}

A fundamental approximation made in existing opacities calculations is to treat autoionizing resonances as 


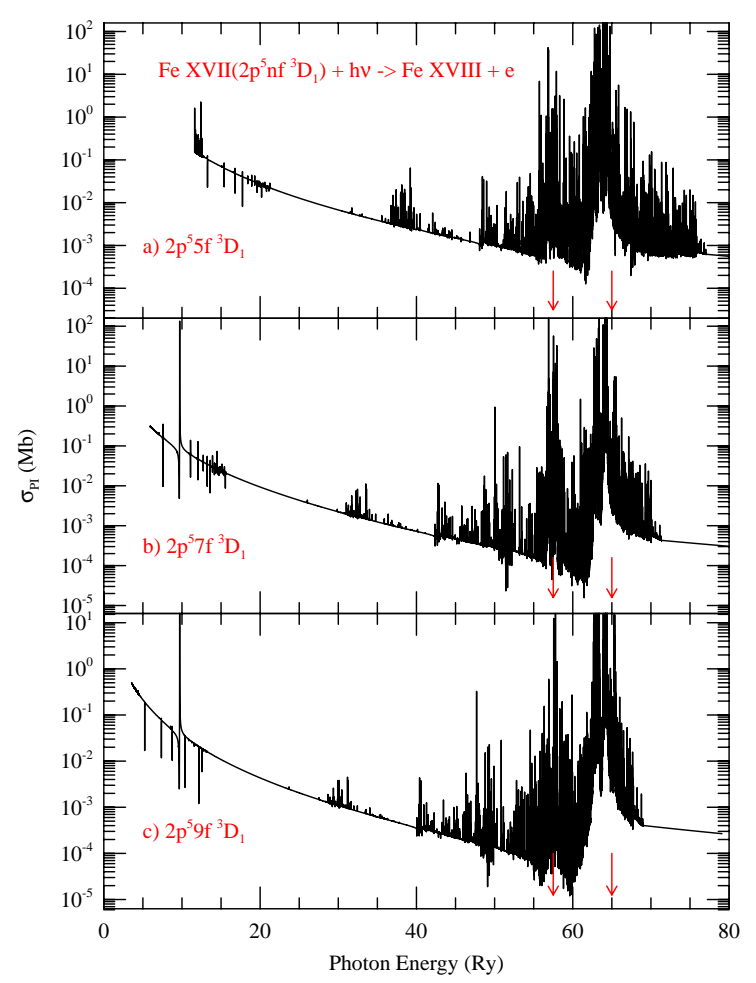

FIG. 3: Total photoionization cross section $\sigma_{P I}$ of highly excited levels of Fe XVII : $2 s^{2} 2 p^{5} n f\left({ }^{3} D_{1}\right)$ with $n f=5 f, 7 f, 9 f$. The cross sections decrease monotonically at lower energies, but rise considerably while approaching the PEC resonances. The figure shows that the cross sections would be enhanced all throughout the energy range, compared to currently available near-hydrogenic cross sections that decrease much faster as $\sigma_{P I} \sim E^{-3}$. The largest PEC resonances are marked by arrows. Although only two such PECs are pointed out, the resonance structures and the resulting enhancement represent the combined effect of all PEC resonances in Table 3. It may be also be noted that the PEC positions remain the same for all Rydberg levels.

lines. In other words, inner-shell excitations leading to bound-free autoionizing states are treated as boundbound transitions. The final state may be further coupled to a feature-less continuum perturbatively to obtain autoionization widths at a single energy associated with the bound-bound transition. This indepndent resonance approximation neglects the intricate coupling effects that are otherwise included via the close coupling method. Since most of the contribution to opacities originates from inner-shell excitations, with final levels as autoionizing states, their impact on opacities bears closer inspection and is briefly discussed below.

In Fig. 4 we examine in some detail one of the cross sections shown in Fig. 3, corresponding to $2 s^{2} 2 p^{5} 7 f\left({ }^{3} D_{1}\right)$.

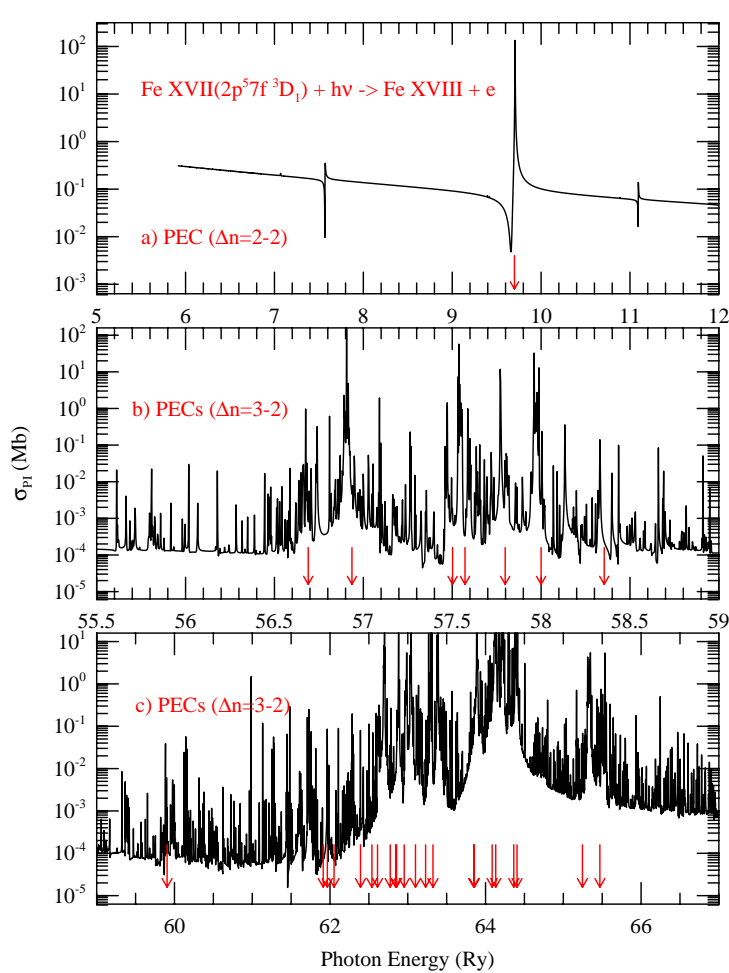

FIG. 4: L-shell PEC resonances in photoionization of Fe XVII . The resonances cover all 60 ionization thresholds included in the CC calculation, including all PEC resonances corresponding to E1 transitions (dipole allowed and intercombination) in Table 3.

The cross sections are delineated at 24,653 energies; the energy intervals are chosen so as to resolve resonance profiles in so far as practical. Postions of all of the PEC resonances due to transitions in Table 3 are marked in Fig. 4 as arrows. In addition, there are a large number of non-PEC Rydberg resonances converging on to the other excited levels. Whereas the few $\Delta n(2-2)$ resonances in Fig. 4a are inconsequential, the myriad $\Delta n(3-2)$ PECs in Fig. 4b and 4c dominate the distribution of oscillator strength over a large region $\sim 56-66 \mathrm{Ryd}$, or $>100 \mathrm{eV}$, due to the L-shell excitation array $2 p \rightarrow 3 d$. The cumulative resonance (or bound-free continuum) oscillator strength corresponding to Fig. 4 is related to the photoionization cross section (viz. 22]) as

$$
\begin{array}{r}
f_{r}\left[\left({ }^{3} D_{1} \longrightarrow \epsilon S L J:^{3}(P, D, F)(J=0,1,2)^{o}\right]=\right. \\
{\left[\frac{1}{4 \pi \alpha a_{o}^{2}}\right] \int_{0}^{\epsilon_{0}} \sigma_{P I}\left(2 s^{2} 2 p^{5} 7 f^{3} D_{1}\right) d \epsilon,}
\end{array}
$$

where $\epsilon$ is the energy relative to ionization threshold and up to $\epsilon_{o}=\sim 80$ Ry. An integration over the range shown in Fig. 4 yields the partial resonance oscillator strength including all of the PECs and the non-PEC resonances 
due to coupling of all $60 \mathrm{Fe}$ XVIII levels. The sum over the oscillator strengths corresponding to the $30 \mathrm{PECs}$ gives a total $f_{P E C}=2.31$. The integrated resonance oscillator strength $f_{r}$ is found to be 4.38 . In other words, the 30 PEC resonances involving transitions up to the $n=3$ levels of the core ion Fe XVIII contribute over half of all the continuum bound-free oscillator strength in photoionization of any excited state of Fe XVII . We note that, without loss of generality, we chose an excited level to demonstrate the quantitative effect of PEC resonances; they manifest themselves in photoionization of most levels.

\section{Monochromatic Opacities}

Opacity calculations are a complex undertaking that requires atomic data for a large number of ions in varying plasma environments [2, 4]. One of the main reasons for the large-scale calculations carried out under the present study is to enable test calculations to benchmark available opacities for Fe ions. To that end, the present BPRM calculations employ similar cut-offs, $n \leq 10$ and $\ell \leq n-1$, as the OP work [3]. However, one usually expects only the ground state and low-lying metastable states to be significantly populated. The ionization fractions and level populations are computed using an equation-ofstate, such as the modified Boltzmann-Saha formulation in the 'chemical picture' [33], based on the premise that isolated atoms exist, albeit perturbed by the plasma environment [2]. At low densities and temperatures the ion fractions and occupation probabilties of high-lying levels are several orders of magnitude smaller than those for the ground state and metastable levels. But in the high temperature-density regime $\mathrm{N}_{e}>10^{24} \mathrm{~cm}^{-3}, \mathrm{~T}_{e}>10^{6}$ $\mathrm{K}$, approaching those in stellar cores, electron-ion recombination rates can be large, and increase rapidly as $\mathrm{N}_{e}^{2}$. Even a small population in excited levels would then be susceptible to the resonant enhancements due to PEC resonances, which are currently neglected and the cross sections for excited levels are taken to be nearly hydrogenic, instead of the accurate form exemplified in Figs. 2 and 3.

While we have discussed integrated resonances oscillator strengths embedded in bound-free cross sections, there is no direct equivalence or 1-1 correspondence with bound-bound oscillator strengths, as generally computed in opacities calculations. Among the factors that distinguish the two are overlapping profiles and large energy widths of PEC resonances, reflecting the coupling of continua belonging to many target levels and strong dipole moments among them. Owing to the huge scale of data needed, definitive checks can only be made by calculating revised opacities using atomic data, as outlined herein.

A practical problem likely to be encountered is the high energy resolution needed to represent resonance profiles. Whereas scattering cross sections are bounded by the unitarity condition (viz. [23], no such upper bound exists for individual values of photoionization cross sections. The peak photoionization values may rise arbitrarily high, and numerical integration would tend to be inaccurate as the resonance profile approaches a Delta function; the integral is finite but the width is extremely narrow and impractical to resolve. However, the statistical methodology adopted in opacities work is to employ the opacity sampling technique. Monochromatic opacity spectra are sampled at approximately 10,000 points (viz. [3]), although the atomic data are much more finely resolved. It has been verified that the statistical averages of the most important quantity, the Rosseland Mean Opacity (RMO), do not deviate by more than $1-3 \%$ even if the atomic cross sections are 'sampled' at $10^{5}$ or $10^{6}$ points [2]. Therefore, the energy mesh of $\sim 30,000$ points used in this work, predominantly in the region of covered by the high energy $n=3$ resonances, should suffice for accurate opacities calculations.

Fig 5 presents the monochromatic opacity $\kappa($ Fe XVII $)$ computed using all of the bound-free data in the present work, 454 photoionization cross sections resolved as mentioned above. The calculations are carried out using a newly developed code for high-precision opacities, adapted from the earlier OP work [2] (the OP opacities are available from the on-line server called OPserver at the Ohio Supercomputer Center [39]). The new code also employs a frequency mesh of $10^{5}$ points, an order of magnitude finer mesh than OP or OPAL, thus obviating some resolution issues in the monochromatic opacity spectra [39]. Essential components of opacities calculations related to bound-bound transitions are retained as in the OP code: electron-impact and Stark broadening, free-free scattering, and electron-photon interaction in the Rayleigh, Thomson, and Compton scattering limits. However, resonance profiles have not yet been broadened. Whereas bound-bound line shapes are symmetrical, the giant PEC resonance profiles span hundreds of eVs (viz. Fig. 3 and 4 ) and are asymmetric. Theoretical formulations for broadening of resonances and algorithms are still being developed. However, as mentioned, plasma effects in the chemical picture [33] are included a posteori, and do not affect the accuray of the atomic parameters computed herein. The details of the new opacities code and results will be reported later. But we note in passing that since the resonances would dissolve more readily than lines, it is likely to significantly affect continuum lowering, manfiest in opacity spectra, and highly senstiive to temperature and density. Lines dissolve and eventually merge into the continuum in high temperature-density regime. Then a precise accounting may not be necessary; they are often treated as "unresolved transition arrays" (UTA) since J-J' transitions are merged together and often subsumed by line broadening [42]. However, our aim is to focus on delineation of atomic features as fully as possible so that their contributions to opacity in different temeperature-density regimes can be accurately ascertained.

The BPRM opacity cross sections in Fig. 5 are com- 


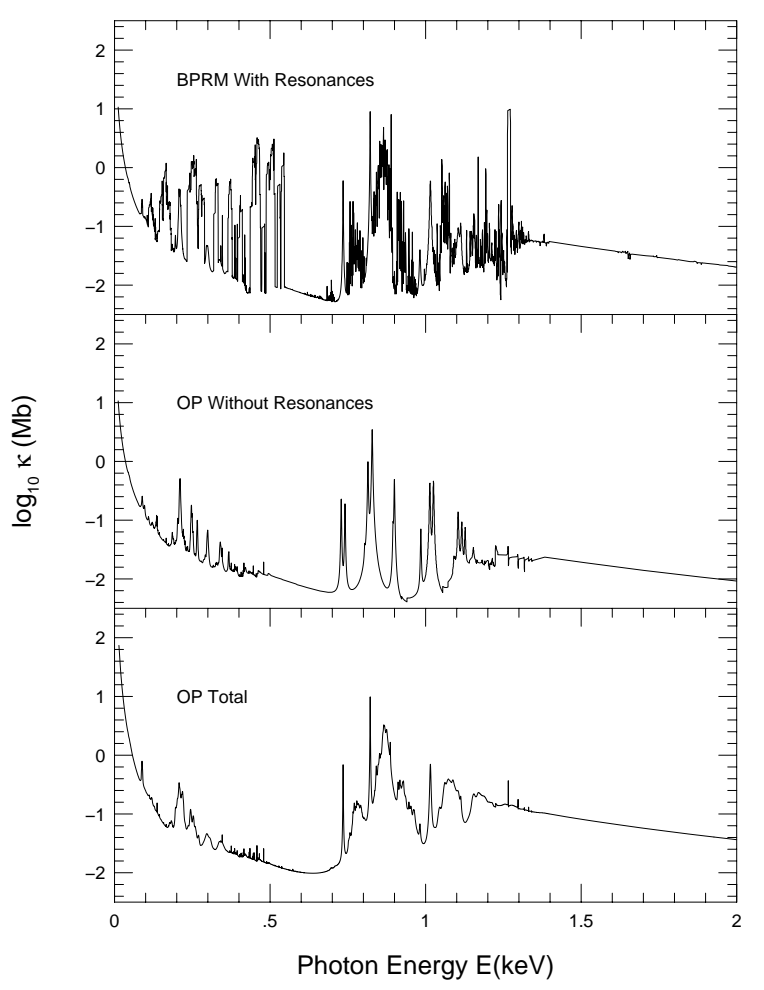

FIG. 5: Partial monochromatic opacity of Fe XVII : $\log _{10} \kappa$ $(\mathrm{Mb})$ at temperature $\mathrm{T}=2.24 \times 10^{6} \mathrm{~K}$ and electron density $N_{e}=10^{23} \mathrm{~cm}^{-3}$, corresponding to the base of the solar convection zone where the Fe XVII ion is the largest contributor to opacity. The top panel presents results using BPRM boundfree cross sections with resonances computed in this work, and the middle panel without resonances using earlier data from the Opacity Project (OP). Identical datasets from OP are employed for the bound-bound transitions, therefore the differences are mainly due to the resonances included in the present work. For comparison, the bottom panel shows total OP opacities [39] that include core-excitation resonances as lines, with autoionization widths considered perturbatively, as well as the high-energy K-shell continuum opacity not yet included in BPRM calculations (see text).

pared to two other sets of results from OP. Although over 20,000 oscillator strengths for bound-bound transitions are also computed, we use exactly the same data for lines as the earlier OP calculations described in [2]. Thus the differences between the the 60CC BPRM results from the present work in the upper panel of Fig. 5, and the limited OP without resonances in the middle panel, are entirely due to the differences in the bound-free data sets. The opacity calculations are done at a temperaturedensity representative of the plasma conditions at the base of the solar convection zone: $\log _{10} \mathrm{~T}(\mathrm{~K})=6.35$ and $\log _{10} \mathrm{~N}_{e}=23$. These parameters also lie in the range currently under investigation in the Z-pinch plasmas for measurment of transmission spectra [1]. In addition to resonance contributions, there are some other differences. The OP data include extrapolated cross sections out to very high energies, $\sim 500 \mathrm{Ry}$. The BPRM data have also been processed to include these high-energy "tails", but the form is slightly different. The background opacity is important to obtain a value for RMOs that spans over 4 decades in temperature, $\log _{10} \mathrm{~T}(\mathrm{~K})=3.5-7.5$.

Calculations for the monochromatic opacity $\kappa_{\nu}$ are carried out for each ion along isotherms in $\log _{10} \mathrm{~T}$ for a range of electron densities $\log _{10} \mathrm{~N}_{e}$. The Rosseland Mean Opacity (RMO) $\kappa_{R}$ is defined in terms of $\kappa_{\nu}$ as

$$
\frac{1}{\kappa_{R}}=\frac{\int_{0}^{\infty} g(u) \frac{1}{\kappa_{\nu}} d u}{\int_{0}^{\infty} g(u) d u}, \quad g(u)=u^{4} e^{-u}\left(1-e^{-u}\right)^{-2},
$$

where $g(u)$ is the Planck weighting function (corrected for stimulated emission). The $\kappa_{\nu}$ is primarily a function of the oscillator strengths $f$, photoionization cross sections $\sigma_{\nu}$, level populations $N_{i}$, and the line profile factor $\phi_{\nu}$,

$$
\kappa_{\nu}^{b b}(i \rightarrow j)=\left(\frac{\pi e^{2}}{m_{e} c}\right) N_{i} f_{i j} \phi_{\nu}, \quad \kappa_{\nu}^{b f}=N_{i} \sigma_{\nu} .
$$

Whereas further code developments are required to include all opacity contributions, we already find large enhancements in $\kappa_{R}$ due to resonances, primarily from the $n=3$ complex. The resonance contribution is included in existing opaciites codes as inner-shell bound-bound transitions [40]. The BPRM value of $\kappa_{R}$, also including the bound-bound oscillator strengths computed in this work (as opposed to OP) yields a value of $223.8 \mathrm{~cm}^{2} / \mathrm{g}$. Using the same bound-bound data as OP, the BPRM $\kappa$ value is still $200.3 \mathrm{~cm}^{2} / \mathrm{g}$, compared to the OP value of $109.7 \mathrm{~cm}^{2} / \mathrm{g}$ (Fig. 5). The bottom panel in Fig. 5 is the total monochromatic opacity spectrum of Fe XVII from $\mathrm{OP}$, including all contributions, with the total RMO $\kappa_{R}$ $=306.9 \mathrm{~cm}^{2} / \mathrm{g}$. Thus the BPRM value using the data computed in this work is $27 \%$ lower. This is primarily because of two factors: (I) The high-energy "tails" are to made more precise with even more extended BPRM calculations including the K-ionization thresholds, which would attenuate the bound-free opacity in the large energy range between the $\mathrm{L}$ and the $\mathrm{K}$ levels. Although the $\mathrm{K}$-shell opacity is not too significant, the K-shell resonances would thereby be included. (II) The bound-bound oscillator strengths are computed up to $n=10$. Therefore, there is a relatively small gap in cross sections in the region between $n=10-\infty$, below the photoionization thresholds of the 454 bound levels. In the earlier OP work, we began the tabulation of cross sections at $\mathrm{E}$ $=-z^{2} / \nu^{2}(\nu \sim 10)$ below each threshold. Employing a similar approximation increases the BPRM RMO value from $223.8 \mathrm{~cm}^{2} / \mathrm{g}$ to $260.7 \mathrm{~cm}^{2} / \mathrm{g}$, to within $20 \%$ of the total OP RMO. Owing to the signficance of (I) and (II), we plan to carry out more extensive calculations than in earlier works, as well investigate if higher- $n$ resonance complexes with $n=4$ or 5 might be needed. 


\section{E. Unified Electron-Ion Recombination}

One of the important charateristics of PEC resonances is that they entail excited states with valence electrons that are weakly bound to the core ion. Therefore, during core excitation the outer electron remains essentially a 'spectator', temporarily attached to the excited core level, and autoionizes as that level decays to the ground state. The analogy and connection between the PEC resonances and the dielectronic recombination process is well known [23, 27, 36]. It is expected that the total electron-ion recombination rates of Fe XVII will also be commensurately enhanced by inclusion of the PEC resonances via detailed balance (Milne relation, e.g. [23, 36]).

A major application of the computed cross sections is in benchmarking total unified recombination rate coefficients, including radiative and dielectronic recombination, for Fe XVII at high temperatures, including those where Fe XVII is abundant in coronal plasmas, such as in the solar corona and solar flares, up to at least $10^{7} \mathrm{~K}$. That is possible because we have considered a large energy range up to the excitation of $n=3$ levels of the recombining ion Fe XVIII . Towards that end, we have also repeated the entire photoionization calculation to obtain partial cross sections for the 454 bound levels of Fe XVII with the residual ion in the ground state alone.

\section{CONCLUSION}

In this report we have presented results from a pilot project of complete BPRM calculations for photoionization of an atomic system, Fe XVII, larger than a He- or Li-like ion, (h $\nu+$ Fe XVII $\rightarrow$ e + Fe XVIII). The aim was to study in detail the extent and range of high energy resonances of importance in practical applications. The BPRM calculations consider all fine structure levels up to $n(S L J) \leq 10$, with spectroscopic identification. In addition to photoionization cross sections, the bound-bound oscillator strengths for transitions among the 454 computed levels of Fe XVII are also being computed. These datasets of atomic parameters should be of unprecedented accuracy and generally useful.

The comprehensive calculations were carried out so as to compare with the erstwhile OP calculations that treated resonances as lines. A clear distinction is made between pure bound-bound transitions and bound-free transitions into autoionizing levels. The results from this work demonstrate that opacities may be computed using BPRM cross sections and transition probabilities, and it is likely that plasma opacities in general, and those of Fe ions in particular, would be different from earlier ones using atomic cross sections that accurately consider the energy distribution of resonances.

Mnochromatic opacities of Fe XVII are computed and compared with the OP work. These are not the final opaciites, and some further developments are still necessary, such as resonance broadening mechanisms and K- shell contributions. Nevertheless, we are able to compute complete BPRM radiative datasets and Rosseland mean opacities that are sufficiently close to OP values to imply that future calculations of plasma opaciites can be carried out with higher precision.

Three other applications of the present work might be pointed out. (i) Calculation of accurate unified electronion recombination rate coefficients. (ii) Benchmarking experimental measurements of absolute photoionization cross sections on accelerator based light sources, that are now being made for multiply charged Fe ions (e.g. 31]). It has now been established that the experimental beams contain ions not only in the ground state but also in several metastable levels. An admixture of ground state plus a few excited metastable levels is therefore necessary to benchmark experimental measurements against theory. In addition, the experimental measurements are capable of reaching energies where PEC resonances occur (e.g. [30]). (iii) The radiative data for Fe XVII should be useful for X-ray spectral diagnostics and non-LTE models ((Ref. 28] presents a detailed Grotrian diagram of $\mathrm{Fe}$ XVII levels up to $n=4$ ).

Estimates of uncertainties in the large sets of parameters reported in this paper, as well as that required in future calculations of opacities, are as follows. The accuracy of the 454 theoretically computed energy levels has been ascertained in the text by comparison with experimentally observed levels, in general better than $1 \%$. The differences between BPRM cross sections and measurements for photoionization and recombination for $\mathrm{Fe}$ XVII are found to be within experimental uncertainties of $\sim 10-20 \%$ 21]. Similarly, most of the strong transition in the extensive dataset of Fe XVII oscillator strengths should be better than 10\% accuracy [41]. The present calculations for photoionization employ a much larger $60 \mathrm{CC}$ eigenfunction expansion than the 3CC calculations in previous studies [21, 41], and are expected to be more accurate. Uncertainites in the BPRM monochromatic opacites should be commensurate with those in the underlying $60 \mathrm{CC}$ data. However, the final accuracy of derived mean Rosseland and Planck opacities ions is as yet undetermined since photoionization cross sections extrapolated or computed at high energies are to be included for all 454 bound states. The problem is further complicated since the aim is to obtain statistical averages with sampled opacities (albeit on a finer mesh of $10^{5}$ frequencies as opposed to $10^{4}$ in earlier calculations) that would approach the accuracy, $\sim 1 \%$, needed to improve over existing uncertainties of $\sim 5 \%$ [19].

Electronic files for photoionization cross sections, energy levels, and oscillator strengths are available electronically from the NORAD website: www.astronomy.ohiostate.edu/ nahar/nahar_radiativeatomicdata 


\section{Acknowledgments}

This work was partially supported by the NASA Astronomy and Physics Research Analysis Program (SNN) and the U.S. Department of Energy (AKP). The computational work was carried out at the Ohio Supercomputer Center in Columbus Ohio.
[1] J. E. Bailey et al. , Phys. Plasmas 16, 058101 (2009).

[2] M.J. Seaton, Y. Yu, D. Mihalas, and A.K. Pradhan, Mon. Not. R. astr. Soc. 266, 805 (1994).

[3] The Opacity Project Team, The Opacity Project, Institute of Physics Publishing, Vol 1, (1995); Vol. 2 (1996).

[4] F. J. Rogers and C. A. Iglesias, in The Opacity Project, Institute of Physics Publishing, Vol. 1 (1995).

[5] D. Grupe, S. Komossa, L. C. Gallo, A. C. Fabian, J. Larsoon, A. K. Pradhan, and D. Xu, Astrophys. J. 681, 982 (2008).

[6] D. Mihalas, Stellar Atmospheres, Freeman (1978).

[7] Rad. Proc. in Physics and Chemistry 70, 323, Elsevier (2004).

[8] P.G. Burke, A. Hibert, and D. Robb, J. Phys. B 4, 153 (1971)

[9] P.G. Burke, W.D. Robb, Adv. At. Mol. Phys. 11, 143214, 1975

[10] P. G. Burke and K. A. Berrington. Atomic and Molecular Processes: an R-matrix Approach (Institute of Physics Publ. Bristol, 1993).

[11] N. S. Scott and K. T. Taylor. Comput. Phys. Commun. 25, 347 (1982).

[12] K.A. Berrington, W.B. Eissner, P.H. Norrington, Comput. Phys. Commun. 92, 290 (1995).

[13] Sultana N. Nahar and Anil K. Pradhan, Astrophys. J. Suppl. 162, 417 (2006)

[14] M. Asplund, N. Grevesse, J. Sauval and P. Scott, Annu. Rev. Astro. Astrohys., 47, 481 (2009).

[15] A. K. Pradhan and S. N. Nahar, in Recent Directions Astrophysical Quantitative Spectroscopy and Radiation Hydrodynamics, AIP Press 1171, 52 (2009).

[16] S. Basu and H.N. Antia, Physics Reports, 457, 217 (2008).

[17] F. Delahaye and M. Pinsonneault, Astrophys. J. 649, 529 (206).

[18] J. N. Bahcall, S. Basu, M. Pinsonneault and A. M. Serenelli, Astrophys. J. 618, 1049 (2005).

[19] M. J. Seaton and N. R. Badnell, Mon. Not. R. astr. Soc. 354, 457 (2004).

[20] A. K. Pradhan, S. N. Nahar, H. L. Zhang, Astrophys. J. Lett. , 549, L265 (2001).

[21] H. L. Zhang, S. N. Nahar, A. K. Pradhan, Phys. Rev. A , 64, 032719 (2001).

[22] S. N. Nahar, H. L. Zhang and A. K. Pradhan, Phys. Rev. A 63, 060701 (2001).

[23] A. K. Pradhan and S. N. Nahar, Atomic Astrophysics and Spectroscopy, Cambridge University Press (2011).

[24] N.S. Scott and K.T. Taylor, Comput. Phys. Commun. 25, 347 (1982).
[25] S. N. Nahar, M. Montenegro, W. Eissner and A. K. Pradhan, Phys. Rev. A 82, 065401 (2010).

[26] Sugar J. \& Corliss C .Elements: Potassium through Nickel. J. Phys. Chem. Ref. Data 1985;14, Suppl. No. 2; listed in NIST compiled website, http://physics.nist.gov/cgi-bin/AtData/main_asd

[27] Y. Yu and M. J. Seaton, J. Phys. B ,, 20, 6409 (1987).

[28] G. X.-Chen, A. K. Pradhan and W. B. Eissner, J. Phys. $B$ 36, 453 (2003).

[29] W. Eissner, M. Jones and N. Nussbaumer, Comput. Phys. Commun. 8, 270 (1974).

[30] M. Habibi, D. A. Esteves, R. A. Phaneuf, A. L. D. Kilcoyne, A. Aguilar A, C. Cisneros, Phys. Rev. A 80, 033407 (2009).

[31] N. El Hassan, J.M. Bizau, C. Blancard, P. Cosse, D. Cubaynes, G. Faussurier, F. Folkmann, Phys. Rev. A 79, 033415-1-8 (2009).

[32] D.G. Hummer, K.A. Berrington, W. Eissner, A.K. Pradhan, H.E. Saraph and J.A. Tully, Astron. Astrophys. 279, 298 (1993).

[33] D. Mihalas, D. G. Hummer and W. Däppen, Astrophys. J. 331, 815 (1988).

[34] Sultana N. Nahar, J. Quant. Spec. Rad. Transfer 109, 2417-2426 (2008)

[35] S.N. Nahar and A.K. Pradhan, Phys. Rev. A 44, 2935 (1991)

[36] S.N. Nahar and A.K. Pradhan, Phys. Rev. A 49, 1816 (1994).

[37] Sultana N. Nahar and Anil K. Pradhan, Physica Scripta 61, 675-689 (2000)

[38] M. J. Seaton, J. Phys. B 20, 6363 (1987).

[39] C. Mendoza, K. J. Seaton, P. Buerger, A. Bellorin, M. Melendez, J. Gonzalez, L. S. Rodriguez, F. Delahaye, E. Palacios, A. K. pradhan and C. J. Zeippen, Mon. Not. R. astr. Soc. , 378, 1031 (2007). The atomic data from the Opacity Project and customized opacities are available from an electronic database - OPserver — at the Ohio Supercomputer Center from http://opacities.osc.edu.

[40] Opacities sources do not usually report monochromatic opacity spectra for individual ions, viz. [39]. Code development is under way to isolate different opacity contributions in the OP work to provide detailed comparisons with improved opacities, using the BPRM data exemplified by the pilot calculations presented herein.

[41] S. N. Nahar, W. Eissner, G.-X. Chen, A.K. Pradhan, Astron. Astrophys. 408, 789 (2003).

[42] B. F. Rozsnyai, S. D. Bloom and D. A. Resler, Phys. Rev. A 44, 6791 (1991), and references therein. 